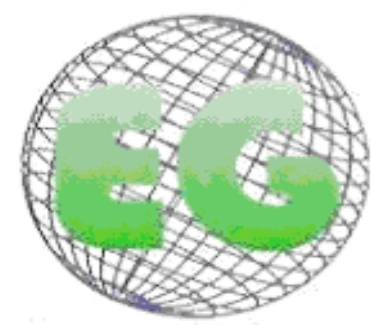

ISSN 1695-6141 N'23

\title{
Salud sexual y reproductiva, anticoncepción e interrupción voluntaria del embarazo en las mujeres inmigrantes latinoamericanas
}

Sexual and reproductive health, contraception and voluntary interruption of pregnancy in women Latin American womens immigrants.

*Rodríguez Portilla, NE., Martínez Rojo, C.

*Matronas del Hospital Universitario Virgen de la Arrixaca. Murcia.

Palabras clave: Salud sexual y reproductiva; Anticoncepción, Interrupción voluntaria embarazo; Mujer; Inmigrante; Planificación familiar; Cultura.

Keywords: Sexual and reproductive health; Contraception; Voluntary termination of pregnancy; Women immigrants; Family planning; Culture.

\section{RESUMEN}

La feminización de la inmigración durante los últimos años y la asociación de esta con el aumento de las interrupciones voluntarias del embarazo es un hecho que está provocando que el Gobierno y los sistemas de salud de cada comunidad se estén preguntando en qué medida los programas de salud sexual y reproductiva y los profesionales sanitarios implicados en estos programas están adaptados a la realidad socio cultural que viven las mujeres inmigrantes.

Esta investigación tiene como objetivo determinar qué factores influyen en el acceso de las mujeres inmigrantes y especialmente las mujeres del colectivo latino a la planificación familiar y programas de salud reproductiva y las circunstancias en las que se producen las interrupciones voluntarias del embarazo en este grupo de mujeres haciendo énfasis en el peso de la herencia cultural que las acompañará durante su proceso migratorio y que será determinante a la hora de afrontar su salud.

\section{ABSTRACT}

The feminization of immigration in recent years and the association of this with the increment of the voluntary termination of pregnancy is a fact that is causing that the government and health systems of each community be wondering what extent health programs sexual and reproductive and the health professionals involved in these programs are adapted to the sociocultural reality experienced by immigrant women. This research aims to determine what factors influence the access of immigrant women, and especially Latin women, in family planning and reproductive health programs and the circumstances in which they opt for voluntary termination of pregnancy with emphasis on the weight of the cultural heritage that accompanies them during the immigration process, and which will be decisive in dealing with their health. 


\section{INTRODUCCIÓN}

Durante los últimos meses la reforma legislativa del aborto es un tema recurrente que está en boca de políticos y sociedad civil. Esta reforma supondrá un cambio sustancial (siempre rodeado de polémica) de los modos y condiciones para la mujer que decide abortar voluntariamente en la España del siglo XXI. La interrupción voluntaria del embarazo pasará de ser un acto despenalizado en ciertos supuestos a ser un derecho de la mujer hasta unas determinadas semanas de gestación.

En España durante los últimos años se ha observado un gran incremento en la notificación de las interrupciones voluntarias del embarazo (IVES), hasta tal punto que en tan solo diez años (entre 1997 y 2007) se ha duplicado el número de dichas notificaciones. Este hecho ha empujado a los investigadores de distintas disciplinas a plantearse cuál es el motivo de dicho incremento y sus consecuencias, poniendo en muchos casos a las mujeres inmigrantes en el "punto de mira" para intentar dar respuesta a dichas cuestiones.

Actualmente la feminización de la inmigración en España es un hecho que está dominando los flujos migratorios en este país durante la última década. Este fenómeno lleva implícito el surgimiento de nuevas necesidades en salud sexual y reproductiva, que deben ser contempladas desde un punto de vista multicultural. Según Pedreño ${ }^{1}$, la feminización de los flujos migratorios se trata de una feminización de la subsistencia donde la mujer ha encontrado en la emigración transnacional una forma de asegurar la supervivencia.

Los factores antes mencionados; la feminización de la inmigración y el aumento de IVES en nuestro país según varios estudios, podrían estar directamente relacionados, de manera que el aumento de inmigración femenina que ha llegado al país en la última década habría traído como consecuencia el incremento en las tasas de interrupciones voluntarias del embarazo, lo que nos hace plantearnos que "algo está fallando" en las políticas y programas de salud reproductiva y sexual que se están llevando a cabo en el país.

En el caso de las mujeres que emigran de su país origen y llegan a España, a pesar de ser un grupo heterogéneo, parece que todas tienen en común multitud de factores que las convierten en un colectivo especialmente "vulnerable" a sufrir embarazos no deseados e interrupciones del embarazo con el consiguiente coste bio-psico-social que supone para estas mujeres. El problema básico al que nos enfrentamos es determinar hasta qué punto el sistema sanitario, los programas y los profesionales tienen en cuenta las creencias culturales que influyen en el concepto de salud que tienen los distintos colectivos de mujeres inmigrantes que conviven en el país, es decir, hasta que punto comprenden qué se entiende por salud en cada cultura y su consistencia hereditaria, término que fue definido por Estes y Zitzow en 1980 como grado en el que el estilo de vida de un individuo refleja su cultura tradicional.

En esta investigación trataremos el tema de la salud sexual y reproductiva, anticoncepción e IVE en la mujer inmigrante en España haciendo especial énfasis en el colectivo latino por dos motivos; por tratarse de un colectivo con una gran representación en este país y por existir un especial contraste entre una cultura tradicional arraigada en su país de origen con los nuevos valores "modernos" de la sociedad española a la que han emigrado.

Este trabajo intenta dar respuestas a nuestra pregunta de investigación: ¿Cuáles son los factores bio-psico-socio- culturales que influyen en el acceso de las mujeres inmigrantes y más concretamente las mujeres del colectivo latino a la planificación familiar y programas de 
salud reproductiva y las circunstancias en las que se producen las interrupciones voluntarias del embarazo en este grupo de mujeres?

En primer lugar realizaremos una contextualización de nuestro tema de estudio mediante el análisis de la situación actual de la inmigración y de la interrupción voluntaria del embarazo en España, con especial mención como señalamos anteriormente, al colectivo latinoamericano. En segundo lugar describiremos las investigaciones realizadas por otros autores sobre el acceso a los programas de salud sexual y reproductiva, la influencia sociocultural en la planificación familiar y el uso de anticonceptivos de las mujeres latinas, la magnitud de los abortos inducidos en inmigrantes y en el colectivo de mujeres latinoamericanas y por último la influencia socio-culturales en la interrupción voluntaria del embarazo en este colectivo.

El objetivo último de esta investigación es contribuir al logro de unos "Cuidados Culturales", término que fue definido por Raquel E. Speector ${ }^{2}$ como "culturalmente apropiados, culturalmente sensibles y culturalmente competentes". Todo ello para conocer las dinámicas y características migratorias que fluyen en nuestra realidad social, y con ese conocimiento hacer un acto de reflexividad antropológica para el cambio en sentido integrador de la mujer latinoamericana en la sociedad murciana en todos sus ámbitos, especialmente en salud sexual y reproductiva.

\section{MATERIAL Y MÉTODO}

Se realiza una revisión bibliográfica sistemática de la literatura científica incluyendo los artículos publicados desde el año 2000 hasta el 2009 en las bases de datos Pub-Med, Cochrane Library, Cuiden, Índice Medico Español y Lilacs. La estrategia de búsqueda combinó diferentes palabras clave: Sexual and reproductive health AND immigrant OR contraception, OR family planing OR voluntary termination pregnancy en cualquier campo de registro y sin limitación del idioma. Una vez obtenidas las referencias de los artículos se realizó una revisión de los mismos seleccionando los realmente transcendentes para la investigación.

\section{RESULTADOS}

3.1 Contexto demográfico, socio-económico y cultural de la inmigración en España. El colectivo latinoamericano

En primer lugar, al tratar el tema de la inmigración debemos señalar que como afirma García Borreguero en el capítulo uno de la obra "La Condición Inmigrante" se trata de un fenómeno social y por tanto está simbólicamente construida con representaciones, ideas y cuestiones que influyen en nuestra manera de ver e interpretar al colectivo de inmigrantes, por tanto no llegaremos a entenderla sin tener en cuenta estas ideas que conforman los conceptos que la sociedad española asocia a la inmigración ${ }^{3}$.

En España, a partir de los años noventa y con la aparición de la inmigración, se produce un cambio de orientación, pasando de ser un país de emigración a ser un país que recibe y acoge a una gran cantidad de extranjeros. Este cambio de orientación ha sido atribuido a factores decisivos como la incorporación de España a la Unión Europea, el lento crecimiento de la población española y un bienestar social y calidad de vida que ha actuado atrayendo nuevos flujos migratorios al país ${ }^{4}$ 
Según la Encuesta Nacional de Inmigrantes 2007 publicada por el Instituto Nacional de Estadística, el número total de nacidos en el extranjero mayores de 15 años y residentes en España el 1 de Enero de 2007 arrojaba un total de 4.526 .522 personas, lo que supone un $10 \%$ del total de la población residente en nuestro país, de las cuales el $52,2 \%$ eran varones y el $47,8 \%$ mujeres.

España ha firmado numerosos convenios sobre migraciones con varios países latinoamericanos, el más amplio fue firmado con Ecuador en el año 2004, por el gran volumen de extranjeros procedentes de este país que llegan a España. Este convenio preveía la regulación de inmigrantes laborales y la amnistía de los extranjeros ilegales.

A finales de los años 90 la Republica de Ecuador comienza a gestar una crisis que se verá agravada por conflictos con Perú y por la caída del precio del petróleo ${ }^{5}$, en 1999 esta importante crisis económica y financiera empuja a cerca de 3 millones de ecuatorianos (20\% del total de la población) a emigrar a países como Estados Unidos, España e Italia.

En una investigación que se publicó en el año 2005 que comparaba la inmigración latinoamericana a EEUU con la inmigración latinoamericana a España, ambos grandes países receptores de este colectivo, se afirmaba que las razones que atraían a los inmigrantes latinos a nuestro país era el crecimiento económico del mismo, la falta de mano de obra y las estrechas relaciones hacia América Latina en el marco de la Comunidad de Naciones Iberoamericanas unido a una política de inmigración más restrictiva en EEUU que en España. Al mismo tiempo, un alto porcentaje de inmigrantes latinos en España contaba con el apoyo de redes familiares aquí y recibía una valoración social más positiva que otros colectivos de inmigrantes. El número de niños por mujer entre ecuatorianas y colombianas era casi el doble de la media nacional. Como conclusión de esta investigación se afirmaba que teniendo en cuenta el factor demográfico, las redes familiares y la política de inmigración en España, la población latinoamericana podría llegar a 4 millones de personas en 2050 (el $10 \%$ de la población $)^{6}$.

Las redes familiares ante la salida de un miembro a otro país movilizan recursos y contactos. Posteriormente los ingresos del migrante y remesas enviadas al país de origen servirán para la migración de otros miembros en un futuro que serán animados a tomar esta decisión por los familiares y amigos que ya han migrado. En algunos casos, la decisión de migrar a una zona determinada de un país está más influenciada por las redes sociales y familiares que el inmigrante tenga en esa zona que por las posibilidades económicas de la misma ${ }^{7}$.

Según Stroscio, los inmigrantes ecuatorianos en el momento de su llegada acuden a dos grandes grupos: la familia y los amigos que se encargan de su acogida y de proporcionarle consejos prácticos para su integración. Como espacios públicos que utilizan destacan los locutorios, como fuente de comunicación y de información, las canchas y los espacios libres que les permiten el contacto con otros miembros del colectivo, las tiendas de alimentos ecuatorianos que les permite mantener un lazo con su país de origen y las escuelas e iglesias que les dan la posibilidad de introducirse en la sociedad de acogida ${ }^{8}$.

Tenemos una imagen homogénea del inmigrante, pero la diversidad social y cultural del país de origen dará lugar a diferentes comportamientos y expectativas del inmigrante una vez que llega aquí, sin olvidar la gran influencia que tendrá la zona donde este inmigrante se asiente; por tanto, lo importante es darnos cuenta de que nos encontramos ante un fenómeno dinámico, donde los problemas y prioridades pueden cambiar en cuestión de meses ${ }^{5}$. 


\subsection{Interrupción voluntaria del embarazo en el colectivo latinoamericano}

España es uno de los países con una tasa más baja de IVES con respecto al resto de países europeos. Según el último informe publicado por el Ministerio de Sanidad durante el año $2008^{9}$ se registraron 115.812 IVES, lo que supone un incremento del 3,27\% con respecto al año anterior. Esta cifra nos muestra cierta estabilización frente al incremento del 10,3\% que se produjo en 2007. Las cifras de IVES en menores de 18 años han descendido un 1,27\% con respecto al 2007. En cuanto al tipo de centro, en 2008 solo se practicaron 2.208 IVE en hospitales públicos, apenas un $2 \%$ del total, la inmensa mayoría se realizó en centros privados. Más de la mitad de las interrupciones voluntarias del embarazo se llevó a cabo dentro de las ocho primeras semanas de gestación siendo el riesgo para la salud materna el motivo del $96 \%$ de los casos.

Cuando intentamos conocer el volumen de abortos que se producen en la población inmigrante que reside en España nos encontramos con que la variable país de origen no aparece en el protocolo de notificación de IVES, por lo que surge la primera dificultad para poder conocer la magnitud del problema en esta población; no existen datos oficiales del fenómeno. Algunos estudios que se han realizado en zonas concretas del país señalan que en torno al 40-50\% de los IVES realizados en España se produce en mujeres extranjeras y especialmente en menores de 25 años.

Por primera vez, el informe publicado por el Ministerio de Sanidad sobre la interrupción voluntaria del embarazo en España durante el 2008 incluye datos sobre el lugar de residencia y nacimiento de la mujer. Según estos datos, los IVES en mujeres nacidas y residentes en España suponen el 54,5\% (63211 casos) frente a un 43,6\% de las IVE en 2008 (50.569 del total de IVE) en mujeres procedentes de otros países y residentes en España. Según el reparto de continentes 26.538 IVES se produjeron en mujeres procedentes de América del Sur ${ }^{9}$.

Por tanto teniendo en cuenta estos datos parece que existe una relación directa entre inmigración y la dificultad del colectivo al acceso de los métodos anticonceptivos y a los servicios sanitarios con un elevado índice de IVES ${ }^{10}$. Un hecho a tener en cuenta que influye de manera decisiva en el fenómeno es la fecundidad del colectivo inmigrante en sus países de origen. Según la Encuesta Nacional de Estadística de 2007 aunque la fecundidad en los países de nacimiento sea, en términos generales, mucho más alta que la de España, parece que la fecundidad de las mujeres nacidas en el extranjero que ya residen en España se parecerá cada vez más a la fecundidad de las mujeres autóctonas ${ }^{11}$.

\subsection{Acceso a los programas de salud sexual y reproductiva del colectivo latinoamericano}

En primer lugar debemos señalar que la sexualidad está más allá de un mero proceso biológico, la sexualidad es una construcción social inmersa en una cosmovisión donde las prácticas y manifestaciones están influenciadas por la cultura y el proceso de socialización de los individuos.

La gran mayoría de estudios realizados sobre el acceso a los programas de salud sexual y planificación familiar parten de la hipótesis de que existen múltiples factores en torno a las mujeres inmigrantes que ponen obstáculos para que estas puedan acceder a estos programas y a unos métodos anticonceptivos eficaces.

Un estudio realizado en Madrid en 2005 sobre conocimiento y uso de anticonceptivos en los inmigrantes latinoamericanos indica que la falta de información y bajo nivel educativo de la 
población, así como la difícil accesibilidad a los métodos, son barreras que afectan especialmente al colectivo de inmigrantes ${ }^{12}$.

La Dra. Serrano ${ }^{13}$ en su artículo "Mujeres inmigrantes y salud reproductiva", señala que la feminización de la inmigración lleva al surgimiento de nuevas necesidades de atención sanitaria en el campo de la salud sexual y reproductiva. Según la autora, la dificultad de adaptar los recursos a las necesidades de estas mujeres se achaca a varios motivos: la salud se sitúa en un segundo plano, desconocen el funcionamiento de la asistencia sanitaria y tiene barreras idiomáticas. A veces están en situación de irregularidad y les resulta incompatible el horario de los centros sanitarios con su trabajo, teniendo miedo a perder el empleo en caso de solicitar faltar a él para acudir al médico. Por último el gasto económico que supone el acceso a determinados métodos anticonceptivos también dificulta su uso.

En el caso de los profesionales, la barrera idiomática y el desconocimiento de otras culturas unido a una gran carga asistencial y rigidez de horarios hace que la asistencia que prestan no se adapte a las necesidades de estas mujeres. En el caso de las mujeres latinas aunque el idioma es un factor integrador de este colectivo, existen términos propios de la herencia cultural referentes a la sexualidad que es fundamental conocer por los profesionales para el préstamo de cuidados culturalmente competentes. Ejemplos de estos términos sería "cuidarse" cuando estas mujeres se refieren al uso de métodos anticonceptivos o "enfermar" cuando se refieren a la llegada de la menstruación.

Con respecto al colectivo de inmigrantes más jóvenes sabemos que existe una mayor falta de información por parte de estas con respecto a los métodos anticonceptivos que en el caso de las jóvenes españolas, en muchas ocasiones como consecuencia del abandono temprano del sistema educativo e integración en el mercado laboral ${ }^{14}$.

En un estudio realizado en Madrid en 2004 mediante entrevistas a profesionales se indicaba como causas del embarazo no deseado el no utilizar métodos anticonceptivos o usar métodos no seguros relacionándolo con el alto precio de estos y la resistencia de los hombres al uso de preservativos. Se resalta la dificultad de acceso a los servicios de planificación familia debido a los horarios incompatibles con su jornada laboral y miedo al despido en caso de ausentarse para acudir a la consulta médica ${ }^{15}$.

$\underline{3.4 \text { Influencias socio-culturales en la planificación familiar y el uso de anticonceptivos de las }}$ mujeres latinas

En primer lugar y antes de comenzar a desarrollar de qué manera influye la cultura en la planificación y anticoncepción , mencionaré que como afirma Rachel E. Spector la dificultad de describir creencias tradicionales radica en el riesgo de caer en estereotipos, prejuicios y discriminación; la herencia cultural y creencias en salud son diferentes en cada grupo étnico y en cada miembro que lo forma, por tanto "dado que cada grupo inmigrante trae consigo actitudes diferentes hacia la enfermedad, la salud y los cuidados de salud, y que incluso dentro de un mismo grupo las creencias y prácticas varían, el conflicto cultural está servido"2.

En 1871 Edward B. Taylor en su artículo "La ciencia de la cultura" definía cultura como "... todo complejo que incluye el conocimiento, las creencias, el arte, la moral, el derecho, las costumbres y cualquiera otros hábitos o capacidades adquiridas por el hombre en cuanto miembro de la sociedad", por tanto nunca llegaremos a conocer cómo y por qué las mujeres son como son y actúan como actúan con respecto a la planificación y el uso de anticonceptivos si no conocemos la carga cultural que han traído de sus países de origen con respecto al tema en cuestión. 
En 2003 el Centro Latinoamericano y Caribeño de Demografía (CELADE) publicó un documento sobre la fecundidad en países latinoamericanos a partir de los factores que $\mathrm{J}$. Bongaart $(1978,1982)$ propone como determinantes de la fecundidad de las poblaciones aumentándola o disminuyéndola: nupcialidad, uso de anticonceptivos, infecundidad postparto y aborto inducido. Según este documento, el inicio de la vida marital en las mujeres de países latinos se inició tempranamente, de manera que el primer matrimonio se produjo en torno a los 20 años en los países estudiados, llegando a este un importante porcentaje de mujeres que ya eran sexualmente activas con anterioridad. El uso de los métodos anticonceptivos en las mujeres latinas ha aumentado en las últimas décadas aunque este documento señala que difiere de unos países a otros, Haití, Guatemala y Bolivia con un uso bajo de anticonceptivos frente a Ecuador, México, Perú, Nicaragua y República Dominicana, donde en el año 2000 más del 60\% de mujeres usaba algún tipo de método para evitar un embarazo.

Parece que en los últimos años los embarazos no deseados entre las mujeres latinoamericanas serían fruto en mayor proporción de un uso incorrecto de los métodos por falta de información que de un desconocimiento de la existencia de dichos métodos. Otros factores que se señalan como determinantes de la planificación familiar serian; el miedo a los efectos secundarios de los anticonceptivos, oposición de la pareja, postura de la iglesia frente a algunos métodos y barreras para acceder a ellos ${ }^{15}$.

Las mujeres latinas que llegan a España siguen conservando su tradición familiar matriarcal. Su sexualidad, su salud sexual y su salud reproductiva son temas concebidos como íntimos, privados, casi secretos, de los que poco o nada se habla; son tabú. Su sexualidad se asocia a algo prohibido salvo para procrear o dar placer y la función primordial de la mujer es la de ser madres. Existe un desconocimiento por parte de estas mujeres de su cuerpo y una falta de información llegando incluso a desconocer cómo se produce un embarazo durante sus primeras relaciones sexuales, lo que desemboca en gestaciones no deseadas poco después del inicio de estas primeras relaciones. Estas mujeres latinas una vez que emigran comienzan a preocuparse por la posibilidad de un embarazo ya que tener un hijo en un nuevo país y en sus circunstancias de inmigrante resultaría muy dificultoso, el problema es que la interiorización y la integración de los métodos anticonceptivos necesitará un tiempo por lo que es frecuente un embarazo no deseado al poco tiempo de llegar a España ${ }^{16}$

En un informe publicado por el observatorio de la mujer se señala que el uso de anticonceptivos entra en conflicto con el modelo tradicional de concepción de la sexualidad del colectivo latinoamericano, la concepción del riesgo como parte del proceso de maduración del joven, la unión de sexualidad y reproducción y considerar los anticonceptivos como "artificiales". El uso de anticonceptivos en el caso de las jóvenes desvela la práctica de relaciones sexuales ante el resto de familiares y amigos siendo catalogadas de "mujeres fáciles" por tanto, en el caso de utilizar algún método les llevaría a decantarse por los más fáciles de ocultar. En el caso del preservativo, su uso se asocia a las relaciones esporádicas, relaciones absolutamente negadas en el modelo de sexualidad tradicional donde priman las relaciones estables ${ }^{14}$.

En una investigación realizada en 2005 sobre conocimientos y uso de anticonceptivos en la población inmigrante latinoamericana en la Comunidad Autónoma de Madrid se determina que todas las personas entrevistadas conocían uno o varios métodos anticonceptivos, siendo el más conocido el preservativo $(97,5 \%)$, seguido de los contraceptivos orales $(95,1 \%)$. El menos conocido fue el diafragma $(36,6 \%)$. La mayoría de los entrevistados recibió la información de los medios de comunicación ${ }^{12}$. 
En un estudio realizado en Barcelona en 2009 sobre salud sexual y reproductiva en inmigrantes bolivianas y ecuatorianas aparece también la idea del proceso de feminización de la inmigración afirmando que tanto entre ecuatorianos/as como entre bolivianos/as las mujeres empadronadas son más que los hombres. En este mismo estudio se afirma que la red de amistades de los inmigrantes favorece el acceso a los métodos anticonceptivos pero que este tipo información resulta insuficiente y con fisuras lo que desemboca en embarazos no deseados y enfermedades de transmisión sexual ${ }^{16}$.

Resultan llamativos los resultados arrojados por una investigación que se realizó entre 19992000 en Madrid sobre las enfermedades de transmisión sexual en inmigrantes que ejercían la prostitución y donde se afirmaba que estas mujeres en el último mes habían utilizado preservativo en el $98 \%$ de las relaciones que habían mantenido con clientes, pero solamente lo habían utilizado en el $17,6 \%$ de sus relaciones privadas ${ }^{17}$.

En el caso de las jóvenes inmigrantes, estas deben hacer un doble proceso de adaptación a los cambios culturales con respecto a la sexualidad, por un lado los cambios propios en la manera de vivir la sexualidad que se produce en todos los adolescentes y por otro lado el cambio que se produce entre la cultura existente en su país de origen y la que deben y pueden ir desarrollando en España ${ }^{10}$.

3.5 La magnitud de los abortos inducidos en inmigrantes y en el colectivo de mujeres latinoamericanas

Para realizar una estimación varios investigadores han realizado estudios de aborto e inmigración en determinadas zonas de España.

En el Distrito Sanitario Levante Alto- Almanzora de Almería durante los años 1998-2002 se determinó que el $52,7 \%$ de interrupciones voluntarias del embarazo se producía entre mujeres inmigrantes de 15 a 49 años, sobre todo en la franja de 20 a 24 años. El aumento de las solicitudes de IVES en esta zona se relacionó con el aumento de población inmigrante $^{18}$.

Un estudio publicado en el Boletín Epidemiológico de la Comunidad de Madrid en 2004 afirma que se estaba produciendo un incremento en el número de IVES cuya causa puede ser que las actuaciones de prevención son insuficientes o que no se adaptan a las necesidades de los colectivos más vulnerables. De los abortos realizados en mujeres residentes de la Comunidad de Madrid en el $52 \%$ de los casos se trataba de mujeres extranjeras con una media de edad de 27 años.

Al analizar el número de IVES en las distintas Comunidades Autónomas se observó que en 2004 las comunidades con una tasa de IVES superior a la media de España eran aquellas que tenían una mayor población inmigrante ${ }^{14}$.

El incremento del número de IVES en España se relaciona con los siguientes aspectos: la mejora en las notificaciones al registro de IVES, al incremento de la población inmigrante y a una mayor fecundidad y número de embarazos no deseados en estas mujeres frente a las españolas $^{10}$. 
Las mujeres en Latinoamérica continúan sufriendo desigualdades de género a pesar de que la situación va cambiando poco a poco en los últimos años, siendo la maternidad y el cuidado del hogar la función destinada a estas mujeres. En América latina el aborto despenalizado solo se practica ante riesgo de muerte de la madre o violación y en algunos países como Chile, El Salvador, Honduras y República Dominicana está prohibido sin excepción, lo que aboca a estas mujeres a la práctica de abortos clandestinos cuyas complicaciones suponen el $21 \%$ de la mortalidad materna a nivel mundial ${ }^{19}$. La clandestinidad de los abortos en su país en cierto modo la reproducen aquí siendo espectacular el número de mujeres inmigrantes que utilizan métodos tradicionales 0 medicamentos que obtienen por Internet y sin prescripción médica para realizar dichos abortos.

En este grupo de mujeres latinoamericanas el aborto estaría relacionado como mencionamos anteriormente con la falta de apoyo familiar por encontrarse esta en su país de origen en la mayoría de los casos y su concepto sobre maternidad y familia que conserva la idea tradicional de que toda responsabilidad de los hijos recae en la mujer comprendiendo que la situación que viven no es idónea para desempeñar su labor de madres ${ }^{16}$.

En el caso de las jóvenes inmigrantes, donde existe una alta tasa de IVES, deben vivir una etapa, la adolescencia, de la que no tiene referencias anteriores en otros miembros de su familia ya que en la mayoría de los casos en sus países de origen pasan directamente de la infancia a la edad adulta pasando por alto la etapa de la juventud que en el caso de España es una etapa larga y por tanto supone un momento de crisis para la creación de vínculos afectivos y sexuales ${ }^{14}$.

El Observatorio de Salud de la Mujer publica un informe sobre la interrupción voluntaria del embarazo en la población adolescente y juventud temprana en el sector inmigrante y concretamente en el colectivo latinoamericano. Analizan el discurso de los inmigrantes mediante grupos de discusión. En estos grupos aparecen dos ideas; la idea de conflicto entre lo tradicional y lo moderno y la idea de pérdida progresiva de los valores tradicionales de su país de origen, aunque al analizar el discurso de los inmigrantes este continúa inserto en el modelo tradicional. El modelo familiar predominante es el de "familia ampliada" y en el discurso de los adultos surgen valores tradicionales como la obligación del matrimonio tras un embarazo, la tendencia a definir todo embarazo como deseado, reforzando así la sexualidad reproductiva, el control comunitario sobre los jóvenes para mantener la institución familiar y la relación estable como la más extendida entre sus hijos.

Con respecto al aborto en el grupo de adultos se produce un rechazo inicial a la práctica del mismo para una evolución posterior más flexible ante determinados casos, siendo la IVE una práctica repudiada pero a la que en ciertos casos se puede recurrir. Los abortos irían en contra de la procreación y de la institución familiar indicando que la familia ampliada será capaz de absorber los posibles problemas que traiga el nacimiento de un miembro más, siendo el IVE un signo de inmadurez por parte del joven. El riesgo de muerte para la madre y de esterilidad tras el aborto es fruto de los resultados que los abortos clandestinos producen en su país de origen, por tanto las experiencias y memoria tanto de la clandestinidad como de las consecuencias de las interrupciones voluntarias del embarazo inevitablemente influirán en su manera de actuar aquí.

En el caso de las jóvenes sudamericanas parecen que están integrando como prioritarios otros objetivos personales y están abandonando el modelo de familia con un gran número de 
hijos como base de su realización personal y social. Por otro lado el proceso de "cortejo" en el que las parejas se conocen en nuestro país se acorta y por tanto se comienzan a mantener relaciones sexuales antes, esto unido a que el uso de preservativo u otros métodos anticonceptivos se asocia en este colectivo a la desconfianza en el otro y a la connotación de "mujer fácil" hace que sean elevadas las prácticas de riesgo entre estos jóvenes. La fuerte desigualdad de género lleva a la presión de los chicos hacia las jóvenes para mantener relaciones sexuales sin preservativo. En el caso de un embarazo no deseado, las parejas se rompen y aunque en un principio aparece un rechazo hacia el IVE posteriormente se acepta anteponiendo el desarrollo personal a la maternidad y ayudado por una mayor permisividad familiar y un sistema sanitario donde la asistencia ofrece mayores garantías que en su país de origen ${ }^{14}$.

Por último decir, que tanto la religión católica, religión a la que pertenece mayoritariamente el colectivo latino, como el resto de religiones influyen de una manera determinante en la percepción de salud y enfermedad de manera que a veces es difícil saber diferenciar que creencias de un individuo derivan de su religión o de la herencia étnica y cultural ${ }^{2}$. La posición que toma la Religión Católica es la de rechazo, tanto a los métodos anticonceptivos exceptuando los métodos definidos como "naturales" como a la práctica de la interrupción voluntaria del embarazo sin excepción.

\section{4- DISCUSIÓN}

En las actuales sociedades globalizadas y diversificadas étnica y culturalmente, encontramos una serie de dificultades para armonizar elementos que conviven recientemente y que dan como resultado una variación del mapa social de nuestra Murcia del siglo XXI. Concretando argumentos, la convivencia del fenómeno inmigratorio (especialmente el femenino) del colectivo latinoamericano y el conocimiento y comprensión de sus conceptos en salud sexual y reproductiva, son fundamentales a la hora de analizar el complejo fenómeno de la planificación familiar y el aborto voluntario en la actual y compleja sociedad española. Solo conociendo y valorando los factores que colocan a la mujer en situación de espacial vulnerabilidad por el hecho migratorio, podemos estimar el alcance de la influencia de la inmigración sobre el aumento de la tasa de abortos en España.

Los números no mienten; el colectivo latinoamericano es el más representado en las estadísticas de IVE en inmigrantes. Esto, a pesar de suponer un choque con sus tradicionales creencias religiosas católicas, sus modelos familiares extensos y su representación de la sexualidad como elemento reproductivo. ¿Cómo explicamos estas contradicciones culturales? Desde el prisma de los duelos migratorios podríamos lanzar algunas propuestas. La mujer que emigra tiene un plan migratorio del que depende no solo su futuro aquí sino el del resto de familiares que permanecen en su país de origen; la frecuencia de los embarazos no deseados de mujeres latinoamericanas recién llegadas a España, viene a poner en riesgo ese plan migratorio, que es el plan de vida que acaba de iniciar y que está "obligado al éxito".

Por otra parte, la mujer latinoamericana por su proximidad cultural con la mujer española se integra más rápido en sociedad que otros colectivos de inmigrantes, y presenta mayor grado de aculturación en patrones de salud sexual y reproductiva. Además son más conscientes de sus necesidades de salud, y son usuarias de los servicios de salud públicos demandando en mayor medida la atención sanitaria, pero en el caso concreto del uso de métodos anticonceptivos existe una carga cultural y un modelo tradicional que envuelve a la sexualidad de estas mujeres que influye de manera decisiva en la decisión de utilizarlos. Toda esta mezcla de pseudointegración y riesgo migratorio, nos aporta herramientas para el 
estudio del incremento del número de IVE en mujeres latinoamericanas y planteamiento de sus posibles soluciones.

Otras cuestiones esenciales en el análisis del aborto en inmigrantes, lo constituyen aquellas relacionadas con las responsabilidades que el Estado tiene sobre esas tasas incrementadas de IVE. Los informes del Ministerio de Sanidad y el Observatorio de la Mujer y las medidas institucionales propuestas como soluciones son como revelan los números, estériles y asépticas. ¿Es nuestro Estado el responsable del fracaso de las políticas de planificación familiar, o es la sociedad la que hace oídos sordos a los Planes de Salud? ¿Es posible que funcionen las políticas de planificación familiar en el colectivo inmigrante latinoamericano si no funcionan en la población autóctona? ¿Hasta qué punto estos Programas Institucionales integran las necesidades de cada inmigrante asociadas a sus condiciones de integración y valores culturales previos?

Es hora de planteamientos innovadores por parte del Estado en materia de salud sexual y reproductiva, para dar cobertura a su población real y no al imaginario del usuario homogéneo ya caduco. Para ello será prioritaria la formación de los profesionales sanitarios en salud intercultural, proporcionando habilidades para el manejo y comprensión de la población inmigrante. Si no conocemos todas las implicaciones culturales que rodean al fenómeno de la anticoncepción y el aborto no podremos encontrar una solución real al problema.

Solo planificando para una sociedad compleja y diversificada, el Estado conseguiría aproximarse a políticas eficaces en cuanto a planificación familiar y reducción en la tasa de embarazos no deseados.

La gran mayoría de estudios que se aproximan al fenómeno de la anticoncepción e interrupción voluntaria del embarazo llegan al acuerdo de que un mayor acceso a servicios públicos y formación del colectivo femenino inmigrante, serían alternativas factibles para mejorar el conocimiento y uso efectivo de métodos anticonceptivos, pero en la realidad, pocas son las propuestas concretas que se llevan a cabo para hacer efectiva dicha accesibilidad y pocos son los programas o políticas que hacen uso del capital social y la redes familiares en las que se apoya la mujer inmigrante latinoamericana para formar y difundir la importancia del buen uso de los anticonceptivos y con ello lograr una reducción de la tasa de IVE. Conocemos las barreras, pero ¿dónde están las soluciones reales al problema?

Como reflexión final podemos decir que solo desde una visión integral de la mujer inmigrante, desde una aproximación multidisciplinar, se puede conocer el fenómeno de la planificación familiar y la interrupción voluntaria del embarazo y que solamente con actividades reales, concretas y factibles, derivadas de ese conocimiento seremos capaces de transmitir a estas mujeres la importancia de la salud sexual y reproductiva en sus vidas.

\section{5- CONCLUSIONES}

La dificultad del acceso de las mujeres inmigrantes a los programas de salud sexual y reproductiva y planificación familiar es un problema multicausal en el que confluyen factores biológicos, sociales y culturales que inciden en el colectivo inmigrante por la situación de vulnerabilidad en la que se encuentran. En el caso del colectivo latinoamericano, la influencia cultural de su país de origen con respecto al uso de anticonceptivos y la interrupción voluntaria es determinante a la hora de afrontar la salud sexual y reproductiva de las mujeres. 


\section{BIBLIOGRAFÍA}

(1) Pedreño A. Sociedades etnofragmentadas. La condición de inmigrante. Exploraciones e investigaciones desde la Región de Murcia. 1ํeㄹición ed. Murcia: Universidad de Murcia publicaciones.; 2005. p. 76-100.

(2) Spector RE. Las culturas de la salud. 2002.

(3) García Borreguero I. La construcción social de la inmigración: el papel de la Universidad. La condición de inmigrante. Exploraciones e investigaciones desde la Región de Murcia. $1^{\circ}$ edición ed. Murcia: Universidad de Murcia Ediciones; 2005. p. 19-32.

(4) Espín G, Ma J. «Inmigración reciente en la Región de Murcia». Papeles de Geografía 2002; 36: 81-104.

(5) Ponce Leiva P. La inmigración ecuatoriana en España: nuevas vidas, nuevos problemas. Acta del 11ํㅡㄹ Congreso de la Asociación Española de Americanistas 2006;1: 223-231.

(6) Gratius S. El factor hispano: los efectos de la inmigración latinoamericana a EEUU y España. Documento de Trabajo (DT) 2005; 49.

(7) Herrera G, Espinosa MCC, Torres A, Migración P. La migración ecuatoriana: transnacionalismo, redes e identidades. : Flacso-Sede Ecuador; 2005.

(8) Stroscio R. Aproximación a las estrategias de integración de los inmigrantes ecuatorianos en la Región de Murcia. La condición de Inmigrante: Universidad de Murcia publicaciones; 2005. p. 301-310.

(9) Ministerio de Sanidad y Política Social. Interrupción voluntaria del embarazo. Datos definitivos correspondientes al año 2008. 2008.

(10) Ministerio de Sanidad y Consumo. La interrupción voluntaria del embarazo y los métodos anticonceptivos en jóvenes. 2007.

(11) Instituto Nacional de Estadística. Encuesta nacional de inmigrantes 2007. 2007.

(12) Hernando V, Álvarez M, Arriola L, Arroyo S, Ballester E, Bermúdez P, et al. Conocimientos y uso de anticonceptivos en la población inmigrante latinoamericana en la Comunidad Autónoma de Madrid. Boletín Epidemiológico Semanal 2005;13(4/3748):37-40.

(13) Serrano I. Mujeres inmigrantes y salud reproductiva. Mujer y salud 20011 de Octubre del 2001; 1 (1):2-3.

(14) CIMOP (Comunicación, imagen y opinión pública). Estudio sociológico: contexto de la Interrupción Voluntaria del Embarazo en población adolescente y juventud temprana. Observatorio de Salud de la Mujer. Ministerio de Sanidad y Consumo 2005.

(15) Llacér Gil de Ramales A, Morales Martín C, Castillo Rodríguez S, Mazarraza L, Martínez Blanco. El aborto en las mujeres inmigrantes. Una perspectiva desde los profesionales sociosanitarios que atienden la demanda en Madrid. Index de Enfermería 2006; 15 (n.55):1-9.

(16) Starkoff PPC. Migrantes en Barcelona: Salud sexual y salud reproductiva entre los derechos y las experiencias migratorias de mujeres ecuatorianas y bolivianas. 2009.

(17) Belza MJ, Clavo P, Ballesteros J, Menéndez B, Castilla J, Sanz S, et al. Condiciones sociolaborales, conductas de riesgo y prevalencia de infecciones de transmisión sexual en mujeres inmigrantes que ejercen la prostitución en Madrid. Gac.Sanit. 2004;18(3):177-183.

(18) Barroso P, Lucerma M, Parron T. Interrupción voluntaria del embarazo en mujeres de un distrito sanitario de Almería durante el periodo 1998-2002. Revista Española de Salud Pública 2005 julio-agosto 2005;79 (004):493-501.

(19) Lamas M. El aborto en la agenda del desarrollo en América Latina. Perfiles latinoamericanos 2008(31):65. 


\section{Anexo 1: MENOPAUSE RATING SCALE (MRS)}

\begin{tabular}{|c|c|c|c|c|c|}
\hline \multicolumn{6}{|c|}{$\begin{array}{l}\text { Con el objeto de conocer sus molestias climatéricas le solicitamos responder el } \\
\text { correspondiente. } \\
\text { ¿Cuál de las siguientes molestias siente en la actualidad y con qué intensidad? }\end{array}$} \\
\hline \multirow{3}{*}{$\begin{array}{l}\text { Tipo de molestia. } \\
\text { (Marque la casilla pertinente de cada molestia } \\
\text { con una "X") } \\
\text { Ejemplo, marque en casilla } 0 \text { cuando "no tiene } \\
\text { molestia" y en la casilla } 1 \text { a la } 4 \text { según cómo } \\
\text { sienta la intensidad de la molestia. }\end{array}$} & \multicolumn{5}{|c|}{ ¿Cómo son sus molestias? } \\
\hline & $\begin{array}{l}\text { No siente } \\
\text { molestia }\end{array}$ & $\begin{array}{l}\text { Siente } \\
\text { molestia } \\
\text { leve }\end{array}$ & $\begin{array}{l}\text { Siente } \\
\text { molestia } \\
\text { moderada }\end{array}$ & $\begin{array}{l}\text { Siente } \\
\text { molestia } \\
\text { importante }\end{array}$ & $\begin{array}{l}\text { Siente } \\
\text { demasiada } \\
\text { molestia }\end{array}$ \\
\hline & $\begin{array}{l}0 \\
:-\mathrm{D}\end{array}$ & $\begin{array}{l}1 \\
:-)\end{array}$ & $\begin{array}{l}2 \\
:-\mathrm{I}\end{array}$ & $\begin{array}{l}3 \\
3 \\
:-(\end{array}$ & $\begin{array}{l}4 \\
-((\end{array}$ \\
\hline \\
\hline \multicolumn{6}{|l|}{$\begin{array}{l}\text { 2. Molestias del corazón (cambios inusuales } \\
\text { en el latido del corazón, palpitaciones, } \\
\text { opresión en el pecho) }\end{array}$} \\
\hline \multicolumn{6}{|l|}{$\begin{array}{l}\text { 3. Problemas de sueño (insomnio, duerme } \\
\text { poco) }\end{array}$} \\
\hline \multicolumn{6}{|l|}{$\begin{array}{l}\text { 4. Molestias musculares y articulares } \\
\begin{array}{l}\text { (dolores de huesos y articulaciones, } \\
\text { dolores reumáticos) }\end{array}\end{array}$} \\
\hline \multicolumn{6}{|l|}{$\begin{array}{l}\text { 5. Estado de ánimo depresivo (sentirse } \\
\text { deprimida, decaída, triste, a punto de } \\
\text { llorar, sin ganas de vivir) }\end{array}$} \\
\hline \multicolumn{6}{|l|}{$\begin{array}{l}\text { 6. Irritabilidad (sentirse tensa, explota fácil, } \\
\text { sentirse rabiosa, sentirse intolerante) }\end{array}$} \\
\hline \multicolumn{6}{|l|}{$\begin{array}{l}\text { 7. Ansiedad (sentirse angustiada, temerosa, } \\
\text { inquieta, tendencia al pánico) }\end{array}$} \\
\hline \multicolumn{6}{|l|}{$\begin{array}{l}\text { 8. Cansancio físico y mental (rinde menos, } \\
\text { se cansa fácil, olvidos frecuentes, mala } \\
\text { memoria, le cuesta concentrarse) }\end{array}$} \\
\hline \multicolumn{6}{|l|}{$\begin{array}{l}\text { 9. Problemas sexuales (menos ganas de } \\
\text { sexo, menor frecuencia de relaciones } \\
\text { sexuales, menos satisfacción sexual) }\end{array}$} \\
\hline \multicolumn{6}{|l|}{$\begin{array}{l}\text { 10. Problemas con la orina (problemas al } \\
\text { orinar, orina más veces, urgencia de } \\
\text { orinar, se le escapa la orina) }\end{array}$} \\
\hline $\begin{array}{l}\text { 11. Sequedad vaginal (sensación de genitales } \\
\text { secos, malestar o ardor en genitales, } \\
\text { malestar o dolor en las relaciones } \\
\text { sexuales) }\end{array}$ & & & & & \\
\hline
\end{tabular}

Fuente: Aedo $2006^{3}$ 